\title{
Functional outcome of clavicle fractures treated with open reduction and internal fixation using plate and screws
}

\author{
Kamal K U1, M Gokul Anand², G Pavan Kumar ${ }^{3 *}$, Shaikh Arbab Mohammad ${ }^{4}$ \\ ${ }^{1}$ Assistant Professor, ${ }^{2}$ Associate Professor, ${ }^{3}$ Post Graduate, ${ }^{4} \mathrm{II} \mathrm{I}^{\text {rd }}$ Year MBBS Student, Department of Orthopedics, Aarupadai Veedu Medical \\ College And Hospital, Puducherry - 60740, INDIA. \\ Email: kamal.lec@gmail.com, dr.pkumar.g@gmail.com, gokul.anand@ymail.com, arbabshaikh400@gmail.com
}

\begin{abstract}
Background: Clavicle fractures account for 44-66\% of all shoulder fractures. Clavicle fractures have been classified by Allman based on the fracture location into three groups (I-III). Most of the clavicle fractures can be treated non-operatively with a global agreement of intervention in displaced and complicated fractures only. Surgical interventions like fixation of the fractures using recon plates and locking compression plates (LCPs) are the most popular maneuvers describe in literature. Methods: We prospectively analyzed the functional and radiological outcomes of 60 surgically treated clavicle fractures. Fractures were classified with Allman's classification. Preoperative radiographs were taken. ORIF with LCPs or recon plate fixation was done. All the patients were followed for 6 months. Functional outcome was evaluated with Quick DASH score. Results: We were able to obtain complete anatomical reduction and fixation of the fractures in all our patients with this technique. Clinical and radiological evaluations indicated that the time of union in our patients ranged from 8-16 weeks (12.5 weeks), post-operatively. The average QUICK-DASH score of our patients at 12 and 24 weeks were reported to be 4.25 and 1.70 respectively. 8 patients reported mild complications during follow-up and were treated according to protocol.
\end{abstract}

Key Words: Clavicle Fractures, Internal Fixation Locking Compression Plate, Recon Plate.

*Address for Correspondence:

Dr G Pavan Kumar, Post Graduate - D. Ortho, Department of Orthopedics, Aarupadai Veedu Medical College and Hospital, Puducherry 607403, INDIA.

Email: dr.pkumar.g@gmail.com

Received Date: 03/04/2020 Revised Date: 13/06/2020 Accepted Date: 30/07/2020

DOI: https://doi.org/10.26611/1031533

This work is licensed under a Creative Commons Attribution-NonCommercial 4.0 International License. $(\mathrm{cc})$ ) EY-NC

\begin{tabular}{|l|l|}
\hline \multicolumn{2}{|c|}{ Access this article online } \\
\hline Quick Response Code: & Website: \\
\hline & www.medpulse.in \\
& \\
\hline
\end{tabular}

\section{INTRODUCTION}

The clavicle is the first bone in the body to begin intramembranous ossification directly from mesenchyme during the fifth week of foetal life. It is a bony attachment with a S-shaped contour providing osseous continuity between the upper limb and thorax. The contouring allows it to serve as a strut for the upper extremity, while also protecting and allowing the passage of the axillary vessels and the brachial plexus medially. The change of contour, which is most acute at the junction of the middle and outer thirds, may explain the frequency of fractures seen in this area. ${ }^{1}$ Given its relative size and the potential force it transmits from the upper limb to the trunk, it is not surprising that it is often fractured. Clavicle fractures are very common injuries in adults $(2-5 \%)$ and children (10$15 \%$ ) and represent about $44-66 \%$ of all shoulder fractures. $^{2}$ The incidence in males is twice as common as that in females. Females show higher prevalence in the sixth decade of life as a result of osteoporosis. ${ }^{3}$ It has been found that the clavicle most often fails in direct compression from force applied directly to the shoulder Direct blow, fall onto an outstretched hand, Moderate to high-energy mechanisms such as motor vehicle accidents or sports injuries in young individuals and a sequel of a 
low-energy fall in elderly individuals leads to clavicle fracture. ${ }^{[4]}$

\section{METHOD AND MATERIALS}

This is a prospective study done in the Department of Orthopaedics at Aarupadai Veedu Medical College and Hospital from July 2018 to January 2020. The total number of cases included in the study was 60 .

- Inclusion criteria -

1. Age more than 18 years and less than 65 years

2. Fracture displacement more than 2 $\mathrm{cm}$

- Exclusion criteria -

1. Age less than 18 years or more than 65 years

2. Pathological fracture

3. Previous or concomitant lesion of the ipsilateral shoulder

4. Neurovascular injury association

5. Open fractures

6. Bilateral fractures

7. Floating shoulder

8. Clinical contraindication to surgery

9. Patient's inability to attend followups,

A detailed clinical history and evaluation including routine blood investigations and proper radiographic evidence with $\mathrm{X}$ - Ray of chest with bilateral shoulder AP view and 45-degree-cephalic tilt view of the involved side was done. Allman classification was used to classify clavicle fractures.

Allman Classification-

- $\quad$ Group I- middle $1 / 3$

- $\quad$ Group II - lateral 1/3 (acromial)

- $\quad$ Group III - medial 1/3 (sternal)

A significant revision of the Allman classification was made by Neer. Group II (distal clavicle) fractures were further divided into 3 types based on the location of the clavicle fracture in relation to the coraco-clavicular ligaments.

These are -

Type I fractures - Minimally displaced and occur lateral to an intact coraco-clavicular ligament complex; these fractures may be treated non-operatively and symptomatically. Type II fractures - Occur when the medial fragment is separated from the coraco-clavicular ligament complex; the medial fragment is displaced cephalic by the pull of the sternocleidomastoid muscle, and the distal fragment is displaced caudally by the weight of the upper extremity, with the intact coraco-clavicular ligament complex; the resulting deformity leads to marked displacement of the fracture ends, predisposing this fracture type to a higher prevalence (upto $30 \%$ ) of nonunion. Type III injuries - Minimally displaced or nondisplaced and extended into the acromioclavicular joint (AC); as with type I fractures, these injuries can be treated symptomatically; the development of the late AC degenerative changes can be treated with distal clavicular excision After necessary evaluations a decision to operate was taken. In all the cases, one hour before the skin incision, a prophylactic preoperative intravenous antibiotic was administered. In a modular operating theater, under strict aseptic precautions, under anesthesia the operative procedures were performed. A curvilinear incision was made over the clavicle, centered over the fracture site. Dissection done in layers, fracture site exposed and edges freshened. The reduction of the fracture was done and confirmed under fluoroscopic guidance. Based on the fracture configuration, either superior plating or anterior plating was done using a dynamic compression plate or a recon plate to fix the fracture. All patients were put on standard arm-sling for comfort. The patients were administered intravenous antibiotics post-operatively for 3 to 5 days. Gentle pendulum exercises started from post-op day 2. Sutures were removed on post-op day 12. Patient was followed up at regular intervals until 24 weeks. Sling was continued until radiographic evidence of healing was seen after which the patient was allowed for unrestricted range of motion exercises but not resistance and exercises or sports activities. Once radiographic union was observed, resistance and strengthening activities were begun. Quick DASH score was used to evaluate the results of the treatment.

\section{RESULT}

A prospective review of experience with clavicle fracture fixation was performed. 60 clavicle fracture fixations using locking compression plate (LCP) and recon plate were performed for patients with fresh fractures over a period of 18 months. The patients (40 men and 20 women) ranged in age from 18 to 60 years (mean age of 39). 34 patients were reported to have right clavicle fracture while the remaining 26 cases were of left clavicle fracture. The most common mode of traumas recorded in our study were road traffic accident ( 28 cases), fall from height ( 24 cases), followed by fall from standing height (7 cases). Sports injury was reported as the cause of trauma in one patient. All patients satisfying surgical intervention indications underwent open reduction and internal fixation (ORIF) procedure. After evaluation of the type/site of injury it was determined that 15 patients had displaced medial-third clavicle fractures providing enough indication for intervention. Fixation using recon plate was approved for 12 patients while the remaining 3 patients of this group were cleared for fixation using locking compression plate (LCP). 10 patients having middle-third clavicle fractures were 
identified upon evaluation and segregated in a different group. Further evaluation revealed complications such as skin tenting indicating the necessity of intervention and repair. 8 patients of this group were identified and cleared for fixation using locking compression plate (LCP) while the remaining 2 patients were treated using recon plate. The remaining 35 patients were observed to have lateral $1 / 3^{\text {rd }}$ clavicle fractures and upon evaluation provided enough evidence of complications to require intervention and repair. 28 fractures of this group were treated with locking compression plate while the remaining 7 fractures were repaired using recon plate. A final review of the patients included in our study reported that 48 fractures were treated with locking compression plate (LCP) and 12 fractures were repaired using recon plate. The duration of hospital stay was reported to be 4-12 days (avg. 6.43 days). All patients were available for follow-up appointments at 1, 2, 3 and 6 months. Clinical and radiological evaluation indicated that the time to union in our patients ranged from 8-16 weeks (avg. 12.5 weeks). The average Quick-Dash score of our patients recorded at 12 and 24 weeks was reported to be 4.25 and 1.70 respectively. 8 of our patients reported complications in the periodic follow-up. Superficial infections observed in 2 patients were treated with oral antibiotics for 1 week leading to full recovery. Hardware irritation was reported in 3 patients. Signs of scar hypertrophy were observed in 2 cases. Shoulder stiffness was recorded in 1 patient which due to noncompliance of rehabilitation protocol.

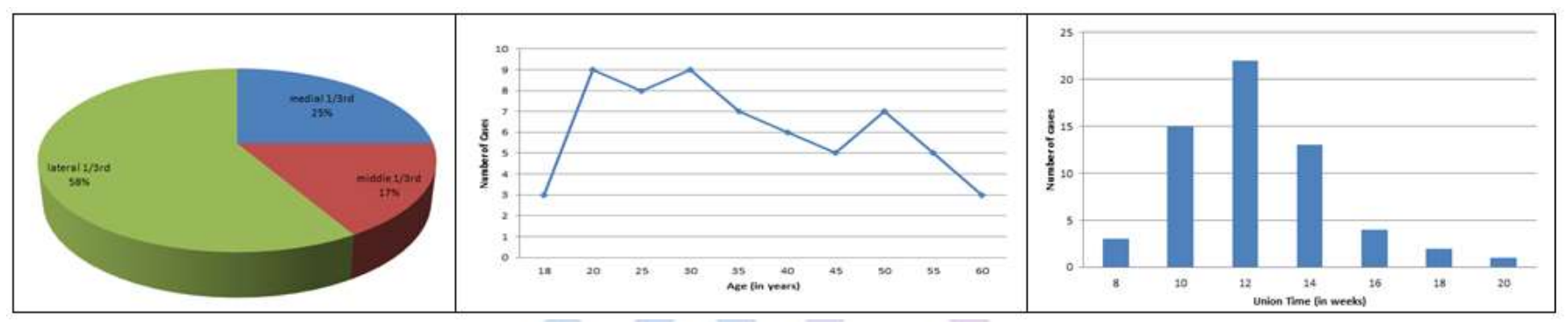

Figure 1: Distribution of cases

Figure 2: Number of cases vs age

Figure 3: Union time(in months) Vs Number of cases

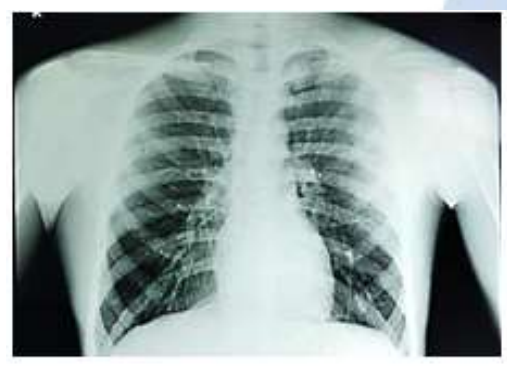

A

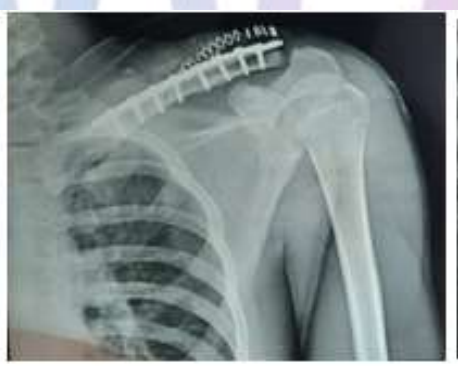

b

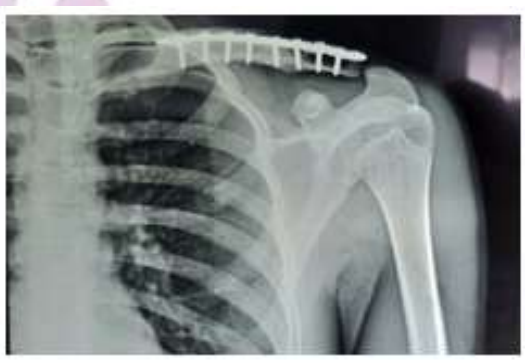

c

Figure 4: Pre operative (a), Post Operative (b) and follow up(c) X-ray.

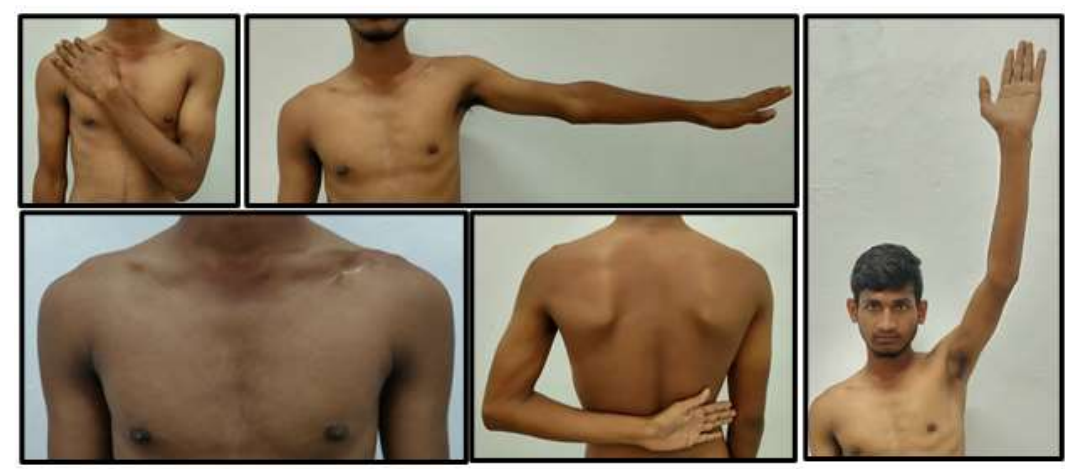

Figure 5: Clinical evaluation at 6 months : Range of motion. 


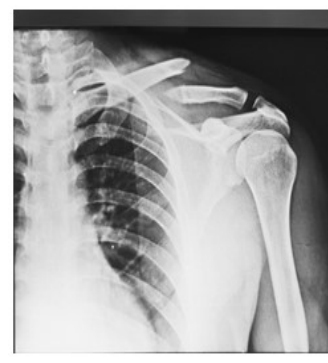

a

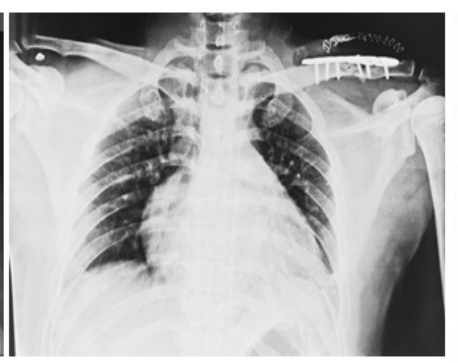

b

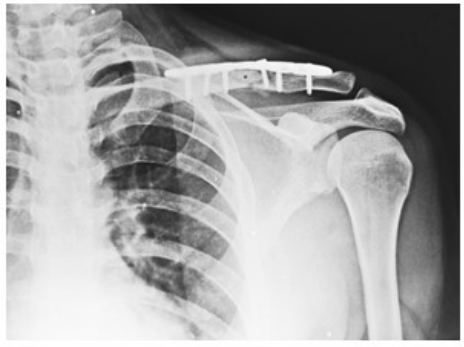

c

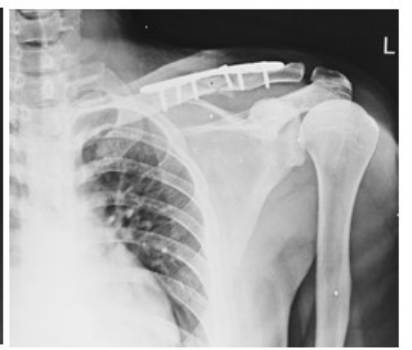

d

Figure 3: Pre op(a), Post op(b) and follow up(c,d) X-rays.

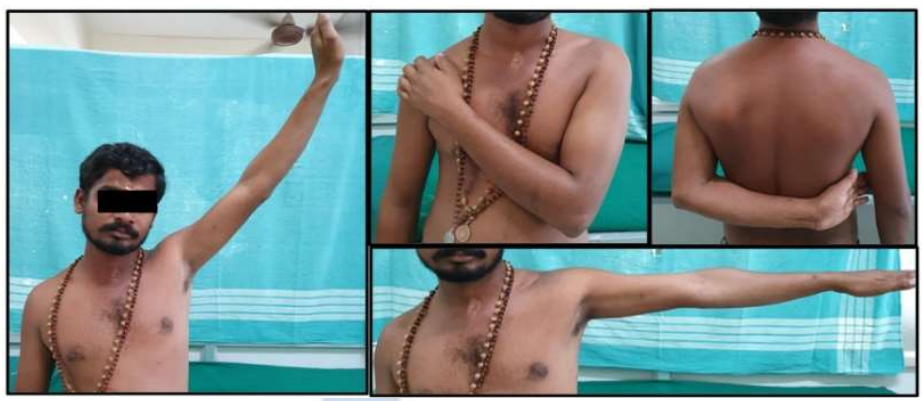

Figure 4: Clinical evaluation at 6 months : Range of motion.

\section{DISCUSSION}

With emergence of refined surgical options and techniques, the management of clavicle fractures has evolved well in the past decade. A personalized treatment that best suits individual patients' activity level is required to

gain satisfactory outcomes in cases of mid-shaft clavicle fractures. Irrespective of segment involved; hand to sling immobilization has time and again proven to be the best possible treatment for non-displaced fractures. Conservative management has been the most popular choice of management of clavicle fractures in India regardless of comminution and displacement. However, many cases of non-union (15\%) have been reported in the literature ${ }^{5}$. It is reported that problems such as malunion, decreased ROM of shoulder and lower satisfactory rate (1/3rd not satisfied) following conservative treatment have made it a less favorable option these days. Studies also describe the wide acceptance of surgical intervention and repair procedures owing to their increased success rates and decreased incidence of malunion as well as non-union complications (only $2.2 \%)^{6}$. Hence, our study primarily focuses on assessment and evaluation of union and functional outcomes post surgical intervention/fixation to treat clavicle fractures. Majority of patients in our study were males(66\%) which makes it comparable to Wun-Jer Shen et al.. (1999) ${ }^{7}$ as well as other similar studies which similarly highlight that the incidence in males is twice as common as in females. H.Jiang et al... study (2012) reports mean time to radiographic union at 12.5 weeks which is comparable with our study findings which report mean time to radiographic union at 12.5 weeks (post-op). In our study, the mean Quick-Dash score, measured at the end of 6 months, is found to be $1,{ }^{7}$ which is in accordance with the finding of a similar study conducted by CM Robinson et al... We have observed hardware problems in 3 patients which amounts to $5 \%$ of the total cases included in our study. The hardware problems encountered can be because the clavicle is a subcutaneous bone with less soft tissue envelope over the implanted hardware contributing to implant-related complications. ${ }^{8}$ Major complications such as neuro-vascular damage, implant leakage, skin necrosis, deep infections or adhesive capsulitis of the shoulder were not seen in our patients. ${ }^{9,10}$ ROM of shoulder was near normal in all our patients post-procedure. The author suggests that it is vital for the surgeon to have a good understanding of the clavicle anatomy and surrounding structures. It is required to employ only those techniques that take into account the anatomy of the clavicle: notably vascularization and acromioclavicular ligament integrity and location of the fracture. It is also important to understand that the plate osteosynthesis should be based on the bone diameter and 3D curvature to avoid post-op complications. Although iatrogenic vascular complications are rare, a good knowledge of vessel relations and variants, especially in the medial end of the clavicle and midshaft improve the procedure outcomes to an extended degree. 


\section{CONCLUSION}

Clavicle fractures have since long been considered as selflimiting injuries mostly treated conservatively. But in the past decade several studies have challenged this claim and according to popular belief surgical intervention to repair comminuted/displaced or complicated clavicle fractures has become widely accepted because of the low incidence in complications and aesthetic disfigurement. Reports of mal-union, non-union and chronic shoulder pain following conservative treatment has contributed to the inclination towards surgical fixation using plates and screws. Internal fixation of these fractures contributes to preservation and restoration of the anatomy while providing stability through implant fixation like locking compression plate (LCP) and recon plate. Plates and screws are favored because of the increased stability they provided and incidence of vascular trauma during surgery is very rare due to minimal contact between the plate and cortical bone. Surgical intervention also helps in speedy recovery with early pain resolution, early union, good ROM, decreased incidence of complications and satisfactory cosmetic results. We observed a reduced rate of complications in our patients (13\%). Minor complications were identified and treated during follow-up resulting in complete recovery. Hence from our study we can conclude that it is a safe and welcoming alternative to conservative treatment with reduced risk of complications.

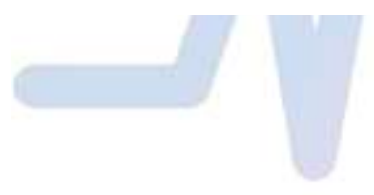

\section{REFERENCES}

1. Postacchini F, Gumina S, De Santis P, Albo F. Epidemiology of clavicle fractures. J Shoulder Elbow Surg. 2002 SepOct;11(5):452-6

2. Canadian Orthopaedic Trauma Society. Nonoperative treatment compared with plate fixation of displaced midshaft clavicular fractures. A multicenter, randomized clinical trial. J Bone Joint Surg Am. 2007 Jan;89(1):1-10.

3. Smekal V, Irenberger A, Struve P, Wambacher M, Krappinger D, Kralinger FS. Elastic stable intramedullary nailing versus nonoperative treatment of displaced midshaft clavicular fractures-a randomized, controlled, clinical trial. J Orthop Trauma. 2009 Feb;23(2):106-12.

4. Wijdicks FJ, Van der Meijden OA, Millett PJ, Verleisdonk EJ, Houwert RM. Systematic review of the complications of plate fixation of clavicle fractures. Arch Orthop Trauma Surg. 2012 May;132(5):617-25. Epub 2012 Jan 10.

5. M. M. Robinson, C. M. Court-Brown, M. M. McQueen, and A. E. Wakefield, "Estimating the risk of nonunion following nonoperative treatment of a clavicular fracture," Journal of Bone and Joint Surgery. Series A, vol. 86, no. 7, pp. 13591365, 2004.

6. Ekegren, Christina L et al... "Incidence, Costs and Predictors of Non-Union, Delayed Union and Mal-Union Following Long Bone Fracture.” International journal of environmental research and public health vol. 15,12 2845. 13 Dec. 2018, doi:10.3390/ijerph15122845

7. Wun-jer Shen, Tsung - Jen Liu, Young - "Shung Shen. Plate fixation of fresh displaced mid shaft clavicle fractures." International journal of the care of the injured. Vol 30, Issue 7, page $497-500$, September 1, 1999.

8. Boehme D et al... Nonunion of fractures of mid shaft clavicle. J Bone Joint Surg (Am). 1991; 73:1219e1226.

9. Mckee MD et al... Midshaft malunions of the clavicle. J Bone Joint Surg (Am). 2003; 85:790e797.

10. Zlowodzki $\mathrm{M}$ et al... treatment of acute midshaft clavicle fractures: systematic review of 2144 fractures; on behalf of the Evidence based Orthopaedic Trauma working group. J Orthop Trauma. 2005; 19:504-7

\section{Source of Support: None Declared} Conflict of Interest: None Declared

\section{Policy for Articles with Open Access:}

Authors who publish with MedPulse International Journal of Orthopedics, (Print ISSN: 2579-0889, Online) (ISSN: 2636-4638) agree to the following terms: Authors retain copyright and grant the journal right of first publication with the work simultaneously licensed under a Creative Commons Attribution License that allows others to share the work with an acknowledgement of the work's authorship and initial publication in this journal.

Authors are permitted and encouraged to post links to their work online (e.g., in institutional repositories or on their website) prior to and during the submission process, as it can lead to productive exchanges, as well as earlier and greater citation of published work. 